\title{
Effect of motor subtypes of delirium in the intensive care unit on fast-track failure after cardiac surgery
}

\author{
Anna Lee, PhD, MPH, ${ }^{a}$ Jing Lan Mu, PhD, ${ }^{a}$ Chun Hung Chiu, MPhil, ${ }^{\text {a }}$ Tony Gin, MD, FANZCA, ${ }^{a}$ \\ Malcolm John Underwood, MD, FRCS (CTh), ${ }^{b}$ and Gavin Matthew Joynt, MBBCh, FCICM ${ }^{\mathrm{a}}$
}

\section{ABSTRACT}

Objective: The purpose of the study was to evaluate the association between motor subtypes of postoperative delirium in the intensive care unit and fast-track failure (a composite outcome of prolonged stay in the intensive care unit $>48$ hours, intensive care unit readmission, and 30-day mortality) after cardiac surgery.

Methods: This was a secondary analysis of a prospective cohort study of 600 consecutive adults undergoing cardiac surgery at a university hospital in Hong Kong (July 2013 to July 2015). The motor subtypes of delirium were classified using the Richmond Agitation Sedation Score and Confusion Assessment Method intensive care unit assessments performed by trained bedside nurses. A generalized estimating equation was used to estimate a common relative risk of fast-track failure associated with motor subtypes.

Results: The incidences of hypoactive, hyperactive, and mixed motor subtypes were $4.3 \%(\mathrm{n}=26), 4.0 \%(\mathrm{n}=24)$, and $5.5 \%(\mathrm{n}=33)$, respectively. Fast-track failure occurred in 88 patients $(14.7 \%)$. There was an association between delirium (all subtypes) and fast-track failure $(P=.048)$; hyperactive delirium (relative risk, 1.95; 95\% confidence interval, 0.96-3.94); hypoactive delirium (relative risk, 2.79; 95\% confidence interval, 1.34-5.84); and mixed delirium (relative risk, 2.55; 95\% confidence interval, 1.11-5.88). Hypoactive and mixed subtypes were associated with prolonged intensive care unit stay (both $P=.001)$.

Conclusions: Patients with pure hypoactive delirium had a similar risk of developing fast-track failure as other motor subtypes. Differentiation of motor subtypes is unlikely to be clinically important for prognostication of fast-track failure. However, because delirium is associated with poor outcomes, potential treatment strategies should address all subtypes equally. (J Thorac Cardiovasc Surg 2018;155:268-75)

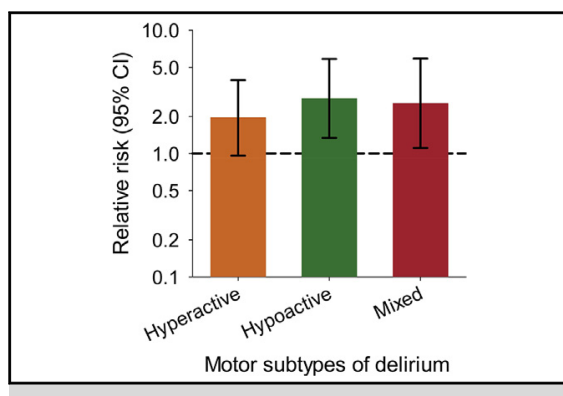

Effect of hyperactive, hypoactive, and mixed ICU delirium on FTF.

\section{Central Message}

Compared with patients without delirium, motor subtypes were more likely to fail fast-track cardiac surgery, with the main contributing factor being a prolonged length of stay in ICU.

\section{Perspective}

We assessed the association between postoperative motor subtypes of ICU delirium and FTF, a composite outcome of prolonged length of stay in ICU, readmission to ICU, and 30-day mortality. Differentiation of motor subtypes is unlikely to be of clinical importance for prognostication in early recovery after cardiac surgery, but treatment strategies should address individual subtypes equally.

See Editorial Commentary page 276.

See Editorial page 240 .
Fast-track cardiac surgery is a complex intervention involving components of standardized perioperative care, with the ultimate aim of early tracheal extubation after cardiac surgery to reduce length of stay in the intensive

From the ${ }^{\mathrm{a}}$ Department of Anaesthesia and Intensive Care, and ${ }^{\mathrm{b}}$ Division of Cardiothoracic Surgery, Department of Surgery, The Chinese University of Hong Kong, Hong Kong, China.

Dr Jin Lan Mu's current address is School of Chinese Medicine, University of Hong Kong, Hong Cong, China.

This research work was fully funded by a grant from the Research Grants Council of Hong Kong Special Administrative Region, China (Project Reference: CUHK469113) and a Direct Grant (2041761) from The Chinese University of Hong Kong.

Institutional Review Board approval: CRE-2012.564, January 20, 2013. care unit (ICU) and the hospital. ${ }^{1,2}$ Fast-track failure (FTF) after cardiac surgery varies widely from $11 \%{ }^{3}$ to $51 \%{ }^{4}$ depending on the definition used, type of cardiac surgery, and age of the patient population studied. In

Received for publication April 25, 2017; revisions received July 6, 2017; accepted for publication Aug 16, 2017; available ahead of print Oct 27, 2017.

Address for reprints: Anna Lee, PhD, MPH, Department of Anaesthesia and Intensive Care, The Chinese University of Hong Kong, 4th Floor, Main Clinical Block and Trauma Centre, Prince of Wales Hospital, Shatin, New Territories, Hong Kong, China (E-mail: annalee@cuhk.edu.hk). $0022-5223 / \$ 36.00$

Copyright (C) 2017 by The American Association for Thoracic Surgery https://doi.org/10.1016/j.jtcvs.2017.08.139 

Abbreviations and Acronyms
AUROC = area under the receiver operating characteristic curve
CABG = coronary artery bypass grafting
CAM = Confusion Assessment Method
$\mathrm{CAM}-\mathrm{ICU}=\mathrm{CAM}$ for the intensive care unit
FTF $\quad$ fast-track failure
GEE = generalized estimating equation
ICU = intensive care unit
IQR = interquartile range
RASS $=$ Richmond Agitation and Sedation Scale
$\mathrm{RR} \quad=$ relative risk

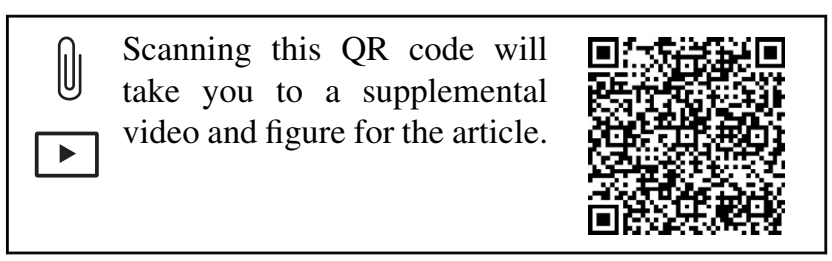

patients after cardiac surgery, FTF is a validated meaningful composite end point. ${ }^{4}$ It incorporates end points that directly and indirectly measure resource use (length of ICU stay), unexpected serious morbidity (readmission to ICU), and meaningful mortality (30-day). Several published risk prediction models for FTF are available, but none include delirium as a candidate predictor. ${ }^{4-7}$

Delirium is a leading postoperative complication and has greater reported effects on adverse outcomes than other major postoperative complications (population attributable risk of $6 \%, 95 \%$ confidence interval [CI], 5-7). ${ }^{8}$ In cardiac surgery, the overall incidence of postoperative delirium ranges from $12 \%$ to $56 \% .^{9-14}$ Patients who develop delirium after cardiac surgery have longer stays in the ICU, higher hospital charges, reduced cognitive and functional recovery, and an increased risk of mortality. ${ }^{12-14}$

It has been suggested that the 3 motor subtypes of postoperative delirium (hyperactive, hypoactive, and mixed) may have differences in underlying pathophysiology, adverse outcomes profile, and prognosis, and may require different pharmacologic management. ${ }^{15-17}$ In a mixed group of surgical patients, those with hypoactive delirium were older and had a higher risk of 6-month mortality than those with mixed delirium (32\% vs $9 \%, P=.04) .{ }^{16}$ Previous studies of motor subtypes of delirium after cardiac surgery suggest that hypoactive delirium is associated with longer duration of mechanical ventilation and length of stay in ICU. ${ }^{17,18}$ However, neither study adequately adjusted for potential confounding bias and mediating effects, or assessed the response to treatment of different subtypes. By understanding the differences in the effect of motor subtypes of delirium on FTF, the underlying predisposing and precipitating factors may be highlighted, a more informative prognosis can be made, and appropriate management strategies considered and tested to potentially achieve positive treatment responses.

The primary objective of this study was to determine the association between motor subtypes of postoperative delirium and FTF after cardiac surgery. A secondary objective was to describe the distribution of delirium subtypes over different sedation levels. We tested the hypothesis that hypoactive delirium was most strongly associated with FTF after cardiac surgery because it is associated with worse prognosis and more adverse events compared with other motor subtypes. ${ }^{16-18}$

\section{MATERIALS AND METHODS \\ Study Participants}

This was a planned secondary analysis of data from a prospective cohort study designed to externally validate and assess the performance of published validated risk prediction models of delirium after cardiac surgery. ${ }^{19}$ The Clinical Research Ethics Committee reviewed and approved the research protocol for the cohort study conducted from July 2013 and July 2015 (CRE-2012.564). All patients gave their written informed consent and were followed up to 30 days after surgery. We recruited 600 consecutive patients undergoing urgent and elective cardiac surgery at The Prince of Wales Hospital in Hong Kong, a 1650-bed university hospital. We excluded patients if they had a sustained Richmond Agitation and Sedation Scale (RASS) score $^{20}$ of -4 or -5 throughout the ICU admission, had major auditory or visual disorders, had mental disabilities, had no CAM-ICU assessment recorded, or were unable to understand Chinese or English. ${ }^{19}$ All patients received standardized perioperative care under existing protocols for anesthesia, surgery, postoperative ICU sedation, analgesia, and weaning from mechanical ventilation. The intraoperative propofol infusion was continued to facilitate transport to the ICU from the operating room and was stopped in the ICU within 3 hours after admission.

\section{Exposure}

We used the Confusion Assessment Method (CAM)-ICU screening tool for detecting delirium. ${ }^{21}$ Trained bedside nurses routinely performed CAM-ICU assessments 3 times per day (once per 8-hour shift) until the patient's discharge from the ICU or up to 7 days during their stay in the ICU, whichever was shorter. Although haloperidol, dexmedetomidine, and quetiapine were prescribed at the discretion of the attending specialist intensivist for the treatment of delirium, all delirium episodes were detected by a positive CAM-ICU tool result.

Motor subtypes of postoperative delirium were classified for CAM-ICU positive results using the corresponding RASS score. ${ }^{20}$ When RASS was scored -5 or -4 , we did not perform CAM-ICU delirium screenings. ${ }^{20}$ Hypoactive delirium was defined if the RASS score was from -3 to 0 , hyperactive if the RASS score was from +1 to +4 , and mixed-type delirium if both hypoactive and hyperactive delirium episodes were present during the observation period. ${ }^{22}$ To reflect the intensity and duration of motor subtypes, we estimated the cumulative delirium severity index ${ }^{23}$ from the CAM-ICU and RASS score assessments recorded in the patient's medical records. The number of delirium exposure days and delirium events was calculated for each patient using the methodology described recently. ${ }^{24}$

\section{Primary Outcome}

The primary outcome was FTF. This is a meaningful patient-centered, objective composite outcome composed of prolonged stay in the ICU 
more than 48 hours, ICU readmission, and 30-day mortality after cardiac surgery. ${ }^{3,25}$ The outcome data were extracted from the Hospital Authority Clinical Management System electronic database.

\section{Other Study Variables}

We collected age, gender, American Society of Anesthesiologists' Physical Status, logistic European System for Cardiac Operative Risk Evaluation, severity of illness score (Acute Physiology and Chronic Health Evaluation II), urgency of ICU admission, type of cardiac surgical procedures, duration of surgery, duration of cardiopulmonary bypass time, perioperative intra-aortic balloon pump support, and duration of mechanical ventilation. We also calculated the predicted risk of delirium derived from the externally validated and calibrated PREdiction of DELIRium in ICu model adapted for this study population. ${ }^{19}$

\section{Statistical Analysis}

Each patient had complete data. Differences in perioperative characteristics between motor subtypes of delirium were compared using Kruskal-Wallis or chi-square tests as appropriate. Spearman's correlation was used to examine the association between the number of delirium-free days and duration of stay in ICU. All statistical tests were performed using Stata 14.2 (StataCorp LP, College Station, Tex).

A generalized estimating equation (GEE) model with a Poisson distribution, log-link function, unstructured correlation, and robust variance was used to obtain a common relative risk (RR) of FTF associated with motor subtypes. The common RR from the GEE model ${ }^{26}$ assumes that there is a single common exposure effect across all components used in the FTF composite end point. The covariates in the GEE model were chosen on the basis of clinical relevance and selected for confounding and mediation adjustments for the causal directed acyclic graph. ${ }^{27}$ These included age, Acute Physiology and Chronic Health Evaluation II score, pharmacologic treatment of delirium, urgency of admission (elective or emergency), perioperative intra-aortic balloon pump support, and duration of mechanical ventilation (Figure E1). Duration of mechanical ventilation (categorized into 3 groups: $<8,8-16,>16$ hours) was deemed a priori as potentially mediating part of the effect of motor subtypes on FTF.

First, we fitted a model by only adjusting for the potential confounders to estimate the "total effect" of motor subtypes through a mediator on FTF using minimal sufficient adjustment sets identified from the DAGitty software. $^{28}$ The total effect was the main analysis chosen because we were interested in the total effect of motor subtypes on outcomes via all pathways. Next, we adjusted for confounding and mediating variables to estimate the "direct effect" of motor subtypes on FTF or the effect "per se" of motor subtypes after accounting for duration of mechanical ventilation using the minimal sufficient adjustment set identified from the DAGitty software. ${ }^{28}$ We assessed whether the magnitude of pure hypoactive delirium was different than other motor subtypes by estimating a $R R$ ratio of pure hypoactive delirium to pure hyperactive delirium groups (hypo/hyper-RR ratio) and of pure hypoactive delirium to mixed delirium (hypo/mix-RR ratio) groups.

We adjusted for multiple testing of individual components of FTF by a Bonferroni correction. For modeling prolonged ICU stay, we used a modified Poisson regression model with robust error variance to estimated the RRs associated with motor subtypes. Because there were too few ICU readmissions and 30-day mortality events, we did not adjust for confounding and mediation and reported the unadjusted RR using Firth logistic regression assuming that the RR is equivalent to odds ratio when events are uncommon. ${ }^{29}$ Model calibration of individual component outcomes was assessed using Hosmer-Lemeshow goodness-of-fit test, but not for assessing models with a composite outcome, because the latter has been shown be problematic because of small cluster sizes. ${ }^{30}$ The bootstrap procedure was used to internally validate the models (1000 samples drawn randomly with replacement of the same size of the original dataset) to estimate a validated area under the receiver operating

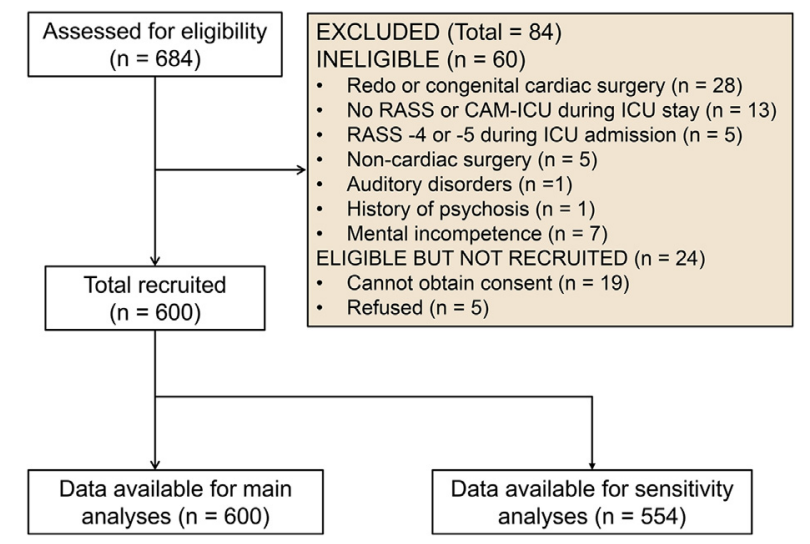

FIGURE 1. Study enrollment summary. RASS, Richmond Agitation and Sedation Scale; CAM-ICU, Confusion Assessment Method for the intensive care unit.

characteristic curve (AUROC) for determining the level of model discrimination.

We conducted a sensitivity analysis because there were concerns about the presence of protopathic bias, that is, patients with a longer duration of ICU stay may be at higher risk of delirium (reverse causation). ${ }^{12} \mathrm{We}$ excluded the delirium exposure in the 8 hours (1 nursing shift) before ICU discharge, carried forward the last delirium exposure status from 8 hours earlier (lag-time approach), ${ }^{31}$ and re-ran the analysis. In this analysis, we excluded some patients who had not yet emerged from coma (RASS -4 or -5 ) in the modified exposure window.

Finally, 6 subtype delirium treatment subgroups (hypoactive no treatment, hyperactive no treatment, mixed no treatment, treated hypoactive, treated hyperactive, and treated mixed) were formed to examine the pharmacologic treatment effect on FTF using a modified Poisson regression model with robust error variance after adjusting for severity of illness and urgency of ICU admission.

\section{Role of the Funding Agency}

The funders had no role in the design and conduct of the study; collection, management, analysis, and interpretation of the data; preparation, review, or approval of the manuscript; and decision to submit the manuscript for publication.

\section{RESULTS}

Of the 684 consecutive patients eligible for the study, 84 were excluded (Figure 1). A total of 46 additional patients were excluded in the sensitivity analyses because they had not yet emerged from coma (RASS -5 or -4 ), a consequence of the introduction of the modified time window, leaving 554 patients. There were $210(35.0 \%)$ coronary artery bypass grafting $(\mathrm{CABG})$ only procedures, $39(6.5 \%)$ CABG-valve procedures, $11(1.8 \%)$ CAGBother procedures, $4(0.7 \%)$ CABG-valve-other procedures, $212(35.3 \%)$ valve-only procedures, $50(8.3 \%)$ valve-other procedures, $59(9.8 \%)$ thoracic vascular procedures, and 15 $(2.5 \%)$ other cardiac procedures. The median (interquartile range [IQR]) duration of mechanical ventilation was 9.1 (5.7-16.8) hours. There was no patient lost to follow-up.

Of the 600 patients, delirium occurred in $83(13.8 \%)$. The incidence of hyperactive $(4.0 \%, 95 \% \mathrm{CI}, 2.6-5.8)$, 


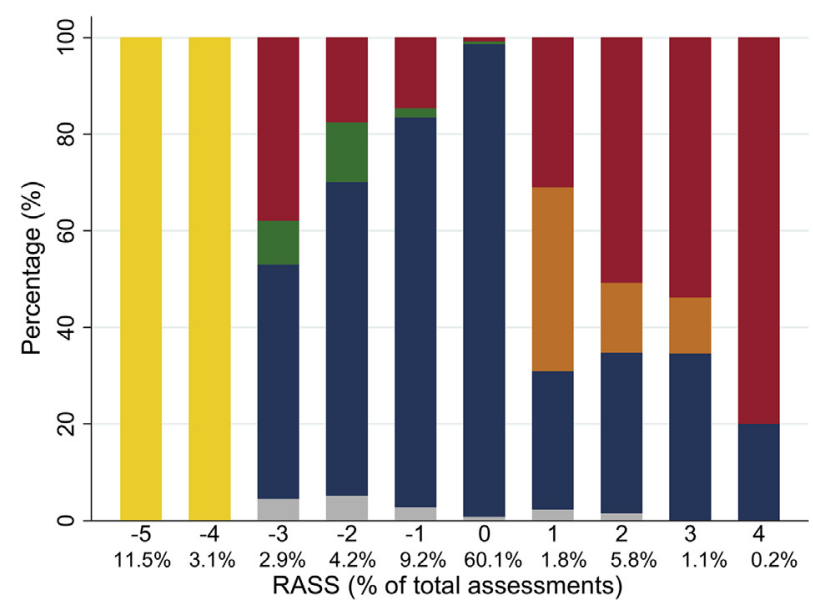

FIGURE 2. Distribution of 2292 motor subtypes of delirium assessments in 600 patients during their stay in the ICU. The percentage of those not assessed because they remained in coma (RASS -4 or -5 ) in yellow, CAM-ICU not done (grey), no delirium (navy), hyperactive delirium (orange), hypoactive delirium (green), or mixed delirium (cranberry) [y axis] by different RASS scores on $\mathrm{x}$ axis. The percentages of total RASS assessments are indicated under each RASS score category. Hypoactive delirium was defined as positive CAM-ICU with a RASS score from -3 to 0 , hyperactive if positive CAM-ICU with a RASS score from +1 to +4 , and mixed-type delirium if both hypoactive and hyperactive delirium episodes were present during the observation period. RASS, Richmond Agitation and Sedation Scale.

hypoactive $(4.3 \%, 95 \% \mathrm{CI}, 2.9-6.2)$, and mixed delirium $(5.5 \%, 95 \%$ CI, 3.9-7.5) was low. The proportion of motor subtypes of delirium ( 24 hyperactive, 26 hypoactive, and 33 mixed) was relatively similar $(28.9 \%, 31.3 \%$, and $39.8 \%$ respectively). The distribution of motor subtypes of delirium episodes at different sedation scores is shown in Figure 2. Although retrospectively diagnosed, the median number of days to the detection of the first episode of delirium (hypoactive or hyperactive) in mixed delirium was longer $(2,1-3)$ than the first episode of pure hyperactive delirium (1, 1-2) and hypoactive delirium (1, 1-1) $(P=.002)$. The differences in perioperative characteristics between motor subtype groups are shown in Table 1.

The protocol stated that postoperative propofol should be stopped within 3 hours. The cessation rate (within 3 hours) was similar $(P=.14)$ among the subtypes of delirium: hyperactive $(71 \%)$, hypoactive $(63 \%)$, and mixed $(45 \%)$. The proportion of patients receiving any type of propofol, midazolam, or dexmedetomidine sedation in the first 24 hours of ICU admission was similar among the hyperactive $(100 \%)$, hypoactive $(92 \%)$, mixed delirium $(100 \%)$, and no delirium $(93 \%)$ groups $(P=.22)$.

Pharmacologic agents (haloperidol, dexmedetomidine, quetiapine) were more likely to be prescribed to patients with mixed delirium (25/33) than with pure hyperactive delirium (10/24) (RR, 1.82; 95\% CI, 1.09-3.03) or pure hypoactive delirium (5/26) (RR, 3.94, 95\% CI,
1.75-8.86). The median (IQR) number of delirium-free days was higher in patients treated with pharmacologic agents (1, 0-2 days) than those not treated $(0,0-1.0)$ $(P=.029)$. There was a moderate correlation between the number of delirium-free days and duration of stay in ICU $(\rho=0.68)$ in the 83 patients with delirium.

\section{Overall Fast-Track Failure Analysis}

Eighty-eight patients $(14.7 \%)$ failed fast-track cardiac surgery. There was a strong unadjusted overall association between delirium and FTF (RR, 7.13; 95\% CI, 4.94-10.28), as well as an individual association of motor subtypes of delirium with the risk of FTF (Table 2). After adjustment for confounding factors (total effect analysis), the overall association of delirium with FTF remained significant (RR, 2.48; 95\% CI, 1.29-4.79), as did individual motor subtypes, mainly the result of a prolonged length of stay in ICU (Table 2). There was excellent discrimination for this total effect model (bootstrapped AUROC 0.89, 95\% $\mathrm{CI}_{\text {bias corrected, }}$ 0.85-0.93). Because the total effect analysis is the most informative, a direct comparison of RRs between hypoactive subgroup with hyperactive and mixed delirium was made. There was no difference in hypo/hyper-RR ratio $(1.43,95 \% \mathrm{CI}, 0.75-2.75, P=.28)$ or in hypo/mix-RR ratio $(1.10,95 \% \mathrm{CI}, 0.55-2.19$, $P=.80$ ). Motor subtype delirium treatment subgroups were not associated with FTF despite adjusting for severity of illness and urgency of ICU admission $(P=.11)$.

In the direct effect analysis, the overall association of delirium with FTF was weaker (RR 1.80; 95\% CI, 0.96-3.39). Thus, motor subtypes were not significantly associated with FTF after accounting for potential confounding and mediation (by duration of mechanical ventilation), indicating that motor subtypes may affect FTF by other means, such as the duration of mechanical ventilation, as opposed to having a direct effect. There was excellent discrimination for this direct effect model (bootstrapped AUROC 0.91, 95\% $\mathrm{CI}_{\text {bias corrected, }}$ 0.87-0.94).

\section{Individual Components of Fast-Track Failure}

In the unadjusted analysis, patients with pure hypoactive delirium were less likely than those with mixed delirium to stay in the ICU longer (RR ratio, $0.44 ; 95 \%$ CI, 0.22-0.87; $P=.004$ ) (Table 2). The total effect model demonstrated that patients with hypoactive or mixed delirium were more likely to have a prolonged ICU length of stay than patients without delirium (Table 2). There was excellent discrimination (bootstrapped AUROC 0.89, 95\% $\mathrm{CI}_{\text {bias corrected, }}$ 0.84-0.93) and adequate calibration (Hosmer-Lemeshow test $P=.63$ ). There were no differences in the hypo/hyper-RR ratio $(1.33,95 \% \mathrm{CI}$, $0.38-4.59, P=.66)$ or the hypo/mix-RR ratio $(0.85,95 \%$ CI, $0.38-1.90, P=.70)$. The direct effect of motor subtypes 
TABLE 1. Characteristics and outcomes of the study population by motor subtypes of delirium groups

\begin{tabular}{|c|c|c|c|c|c|}
\hline & $\begin{array}{c}\text { None } \\
(\mathrm{n}=\mathbf{5 1 7})\end{array}$ & $\begin{array}{l}\text { Hyperactive delirium } \\
\qquad(\mathrm{n}=\mathbf{2 4})\end{array}$ & $\begin{array}{l}\text { Hypoactive delirium } \\
\qquad(\mathbf{n}=\mathbf{2 6})\end{array}$ & $\begin{array}{l}\text { Mixed delirium } \\
\quad(\mathbf{n}=\mathbf{3 3})\end{array}$ & $P$ value \\
\hline Age, median (IQR), y & $61.0(54.0-66.0)$ & $65.0(56.5-73.3)$ & $67.0(57.8-76.0)$ & $63.0(51.0-69.5)$ & .004 \\
\hline Males sex & $358(69.2)$ & $15(62.5)$ & $15(57.7)$ & $25(75.8)$ & .44 \\
\hline ASA-PS classification $\geq \mathrm{III}(\mathrm{n}, \%)$ & $406(78.5)$ & $18(75.0)$ & $20(76.9)$ & $29(87.9)$ & .59 \\
\hline Median (IQR) anesthesia duration, $\mathrm{h}$ & $4.6(4.0-5.2)$ & $5.4(4.2-7.0)$ & $4.3(4.0-4.6)$ & $6.2(4.7-8.1)$ & $<.001$ \\
\hline Median (IQR) surgery duration, $h$ & $3.7(3.1-4.3)$ & $4.3(3.2-5.7)$ & $3.3(2.9-3.9)$ & $4.9(3.3-7.0)$ & $<.001$ \\
\hline Median (IQR) CPB time, min & $104(86-140)$ & $137(84-216)$ & $92(82-130)$ & $174(131-248)$ & $<.001$ \\
\hline Median (IQR) logistic euroSCORE (\%) & $3.2(2.0-7.0)$ & $10.9(3.8-38.2)$ & $5.9(3.4-11.9)$ & $19.1(10.3-28.8)$ & $<.001$ \\
\hline Urgent ICU admission (n, \%) & $54(10.4)$ & $7(29.2)$ & $5(19.2)$ & $20(60.6)$ & $<.001$ \\
\hline Median (IQR) APACHE-II score & $13.0(10.0-16.0)$ & $16.0(12.0-20.0)$ & $15.5(12.0-19.3)$ & $17.0(14.0-20.0)$ & $<.001$ \\
\hline Median (IQR) PRE-DELIRIC (\%)* & $9(6-15)$ & $17(9-33)$ & $17(10-24)$ & $22(14-48)$ & $<.001$ \\
\hline Median (IQR) mechanical Ventilation, $h$ & $8.3(5.3-14.2)$ & $16.2(8.4-86.9)$ & $18.5(9.6-41.1)$ & $65.2(19.9-253.8)$ & $<.001$ \\
\hline Median (IQR) No. of delirium episodes & $0(0-0)$ & $1(1-2)$ & $1(1-1)$ & $4(3-8)$ & $<.001$ \\
\hline Median (IQR) duration of delirium, $\mathrm{d}$ & $0(0-0)$ & $1(1-1)$ & $1(1-1)$ & $2(2-4)$ & $<.001$ \\
\hline Median (IQR) delirium severity index $\dagger$ & $0(0-0)$ & $3.0(2.0-4.0)$ & $3.0(1.0-4.0)$ & $10(6.5-23.5)$ & $<.001$ \\
\hline Drug treatment for delirium $(\mathrm{n}, \%)$ & $0(0)$ & $10(41.6)$ & $5(19.2)$ & $25(75.8)$ & $<.001$ \\
\hline Median (IQR) ICU stay, h & $21.8(19.3-23.2)$ & $24.4(21.2-107.4)$ & $27.5(22.2-63.8)$ & $128(60.7-314.2)$ & $<.001$ \\
\hline ICU stay $>48 \mathrm{~h}(\mathrm{n}, \%)$ & $28(5.4)$ & $8(33.3)$ & $9(34.6)$ & $26(78.8)$ & $<.001$ \\
\hline Median (IQR) hospital stay, d & $12.0(9.0-17.0)$ & $16.5(11.3-22.8)$ & $16.5(12.0-30.8)$ & $23.0(13.5-38.5)$ & $<.001$ \\
\hline ICU readmission (n, \%) & $13(2.5)$ & $1(4.2)$ & $2(7.7)$ & $1(3.0)$ & .46 \\
\hline 30-d mortality (n, \%) & $5(1.0)$ & $1(4.2)$ & $1(3.8)$ & $0(0.0)$ & .26 \\
\hline FTF $(n, \%)$ & $41(7.9)$ & $10(41.7)$ & $11(42.3)$ & $26(78.8)$ & $<.001$ \\
\hline
\end{tabular}

$I Q R$, Interquartile range; $A S A-P S$, American Society of Anesthesiologists' Physical Status classification system; CPB, cardiopulmonary bypass; euroSCORE, European System for Cardiac Operative Risk Evaluation; ICU, intensive care unit; APACHE, Acute Physiology and Chronic Health Evaluation; PRE-DELIRIC, PREdiction of DELIRium in ICu patients; FTF, fast-track failure. *Recalibrated for Prince of Wales Hospital, Hong Kong. ${ }^{19} \dagger$ Sum of delirium severity index (reflects intensity and duration).

on prolonged ICU length of stay was significant (Table 2), mainly due to the effect in the mixed delirium group. The direct effect model had excellent discrimination (bootstrapped AUROC $\left.0.93,95 \% \mathrm{CI}_{\text {bias corrected }}, 0.91-0.95\right)$ and adequate calibration (Hosmer-Lemeshow test $P=.55$ ). The associations between motor subtypes and risk of ICU readmission or between motor subtypes and 30-day mortality were imprecise (Table 2).

In the sensitivity analysis, we chose 8 hours for the time-lag interval because the median (IQR) duration of ICU length of stay in 67 patients with early delirium (within 48 hours after surgery) appeared to be shorter than when all 83 patients with delirium were included in the main analysis (43.9 hours, 22.3-90.4 vs 51.1 hours, 22.6-143.9). The sensitivity analysis showed stronger associations between motor subtypes and FTF were present (Table 3). However, none of the RR ratio comparisons was significant in the adjusted analyses. Likewise, there were strong associations between motor subtypes and prolonged ICU length of stay (Table 3). The associations between motor subtypes and risk of ICU readmission, or between motor subtypes and 30-day mortality were imprecise (Table 3 ).

\section{DISCUSSION}

This prospective cohort study showed that 1 in 7 patients had delirium. Compared with patients without delirium, motor subtypes were more likely to fail fast-track cardiac surgery, with the main contributing factor being a prolonged length of stay in ICU. However, there was no difference in the RR estimates among the various motor subtypes groups after risk factor adjustments. The association between motor subtypes and risk of readmission to ICU or 30-day mortality was imprecise because of the low occurrence. The results were robust when considering the possibility of a protopathic (time-lag effect) bias.

The incidence of hyperactive, hypoactive, and mixed delirium in ICU after cardiac surgery reported in the current article is within the range reported by others. ${ }^{12,17,18,32} \mathrm{~A}$ post hoc comparison among motor subtypes showed that patients with mixed delirium spent more time on the ventilator than those with hyperactive delirium (Bonferroni corrected $P=.042$ ) (Table 1). This was probably due to a higher number and longer duration of delirium episodes (ie, severity) in patients with mixed delirium than other motor subtypes (both Bonferroni 
TABLE 2. Common effect of motor subtypes of delirium across fast-track failure components among 600 cardiac surgical patients: Reference group is no delirium group

\begin{tabular}{|c|c|c|c|c|}
\hline Model adjustment & $\begin{array}{c}\text { Hyperactive delirium } \\
(\mathbf{n}=\mathbf{2 4})\end{array}$ & $\begin{array}{c}\text { Hypoactive delirium } \\
(\mathbf{n}=\mathbf{2 6})\end{array}$ & $\begin{array}{l}\text { Mixed delirium } \\
\quad(\mathbf{n}=\mathbf{3 3})\end{array}$ & $\begin{array}{c}\text { Overall } \\
\text { comparison }\end{array}$ \\
\hline$\overline{\text { FTF (composite) }}$ & RR $(95 \%$ CI) & RR $(95 \%$ CI $)$ & RR $(95 \%$ CI $)$ & $P$ value \\
\hline Unadjusted & $4.86(2.74-8.64)$ & $5.37(3.08-9.35)$ & $10.15(7.07-14.59)$ & $<.001$ \\
\hline Total effect* & $1.95(0.96-3.94)$ & $2.79(1.34-5.84)$ & $2.55(1.11-5.88)$ & .048 \\
\hline Direct effect $\dagger$ & $1.49(0.74-2.99)$ & $2.00(1.00-4.03)$ & $1.82(0.81-4.09)$ & .27 \\
\hline Individual components & RR $(98.3 \%$ CI $)$ & RR $(98.3 \%$ CI $)$ & RR $(98.3 \%$ CI $)$ & $P$ value \\
\hline \multicolumn{5}{|l|}{ ICU LOS $>48 \mathrm{~h}$} \\
\hline Unadjusted & $6.15(2.71-13.97)$ & $6.39(2.93-13.96)$ & $14.55(8.91-23.76)$ & $<.001$ \\
\hline Total effect* & $3.08(0.71-13.25)$ & $4.08(1.43-11.68)$ & $4.78(1.76-13.00)$ & .001 \\
\hline Direct effect $\dagger$ & $2.17(0.87-5.37)$ & $2.57(0.94-7.00)$ & $2.97(1.13-7.77)$ & .044 \\
\hline ICU readmission (unadjusted) $\ddagger$ & $2.39(0.29-19.89)$ & $3.81(0.68-21.31)$ & $1.72(0.21-14.15)$ & .24 \\
\hline 30-d mortality (unadjusted) $\ddagger$ & $5.95(0.62-57.09)$ & $5.48(0.57-52.39)$ & $1.39(0.04-48.94)$ & .13 \\
\hline
\end{tabular}

FTF, Fast-track failure; $R R$, relative risk; $C I$, confidence interval; $I C U$, intensive care unit; $L O S$, length of stay. *Adjusted for the following confounders: Acute Physiology and Chronic Health Evaluation II score, pharmacologic treatment of delirium, and urgent admission. †Adjusted for the following confounders: Acute Physiology and Chronic Health Evaluation II score, pharmacologic treatment of delirium, urgent admission, and mediator: duration of mechanical ventilation. The set of variables for adjustments were identified according to the DAGitty software. ${ }^{28} \ddagger$ Because there were too few events for adjustment, we used Firth logistic for sparse events and assumed the odds ratio was equivalent to RR.

corrected $P<.001$ ). In other studies, the severity of delirium was also associated with longer duration of mechanical ventilation, ${ }^{17}$ longer hospital length of stay, ${ }^{12}$ higher hospital costs, ${ }^{12}$ and in-hospital mortality (odds ratio, $1.47 ; 95 \%$ CI, 1.30-1.66). ${ }^{33}$

Hypoactive motor subtype is difficult to recognize clinically because this group of patients show less overt signs of agitation (as occurs in hyperactive and mixed subtypes), and they are easy to nurse. Its significance in prognostication and resource implications compared with other motor subtypes have been unclear, because few studies have directly compared various motor subtypes with robust attempts at adjustment for confounders. In this study, the effect of pure hypoactive delirium was no different than other motor subtypes on FTF or prolonged length of stay in the ICU after risk factor adjustment. In addition, patients with pure hypoactive delirium were more likely to have a prolonged ICU stay than those without delirium, a finding that is consistent with previous studies. ${ }^{12,18}$

Given the low rate of readmission to the ICU and 30-day mortality, we were unable to find a difference in risk among the motor subtypes of delirium. However, a previous study showed that patients with hypoactive delirium were up to 13 times more likely to die within 5 months after cardiac surgery than those without delirium. ${ }^{18}$

TABLE 3. Common effect of motor subtypes of delirium across fast-track failure components among 554 cardiac surgical patients taking into account protopathic bias: Reference group is no delirium group

\begin{tabular}{|c|c|c|c|c|}
\hline Model adjustment & $\begin{array}{l}\text { Hyperactive delirium } \\
(\mathbf{n}=\mathbf{2 3})\end{array}$ & $\begin{array}{l}\text { Hypoactive delirium } \\
(\mathbf{n}=\mathbf{2 6})\end{array}$ & $\begin{array}{l}\text { Mixed delirium } \\
\quad(\mathbf{n}=27)\end{array}$ & $\begin{array}{c}\text { Overall } \\
\text { comparison }\end{array}$ \\
\hline FTF (composite) & RR $(95 \%$ CI $)$ & RR $(95 \%$ CI $)$ & RR $(95 \%$ CI) & $P$ value \\
\hline Unadjusted & $5.61(3.30-9.54)$ & $5.54(3.27-9.39)$ & $10.51(7.35-15.04)$ & $<.001$ \\
\hline Total effect* & $2.66(1.35-5.26)$ & $2.71(1.07-6.89)$ & $3.66(1.94-6.90)$ & $<.001$ \\
\hline Direct effect ${ }^{\dagger}$ & $1.82(0.93-3.55)$ & $1.78(0.75-4.27)$ & $2.41(1.20-4.83)$ & .09 \\
\hline Individual components & RR $(98.3 \%$ CI $)$ & RR $(98.3 \%$ CI $)$ & RR $(98.3 \%$ CI $)$ & $P$ value \\
\hline \multicolumn{5}{|l|}{ ICU LOS $>48 \mathrm{~h}$} \\
\hline Unadjusted & $7.42(3.62-15.24)$ & $6.57(3.14-13.75)$ & $14.54(9.00-23.49)$ & $<.001$ \\
\hline Total effect* & $4.64(2.17-9.93)$ & $4.16(1.30-13.27)$ & $6.81(3.18-14.58)$ & $<.001$ \\
\hline Direct effect $\dagger$ & $3.07(1.33-7.08)$ & $2.47(0.86-7.11)$ & $4.06(1.69-9.77)$ & .002 \\
\hline ICU readmission (unadjusted) $\ddagger$ & $2.49(0.30-20.97)$ & $3.81(0.67-21.50)$ & $2.11(0.25-17.64)$ & .23 \\
\hline 30-d mortality (unadjusted) $\ddagger$ & $1.83(0.05-65.13)$ & $5.06(0.53-48.41)$ & $1.57(0.04-55.38)$ & .39 \\
\hline
\end{tabular}

$F T F$, Fast-track failure; $R R$, relative risk; $C I$, confidence interval; $I C U$, intensive care unit; $L O S$, length of stay. *Adjusted for the following confounders: Acute Physiology and Chronic Health Evaluation II score, pharmacologic treatment of delirium, and urgent admission. $\nmid$ Adjusted for the following confounders: Acute Physiology and Chronic Health Evaluation II score, pharmacologic treatment of delirium, urgent admission, and mediator: duration of mechanical ventilation. The set of variables for adjustments were identified according to the DAGitty software. ${ }^{28} \ddagger$ Because there were too few events for adjustment, we used Firth logistic for sparse events and assumed the odds ratio was equivalent to RR. 


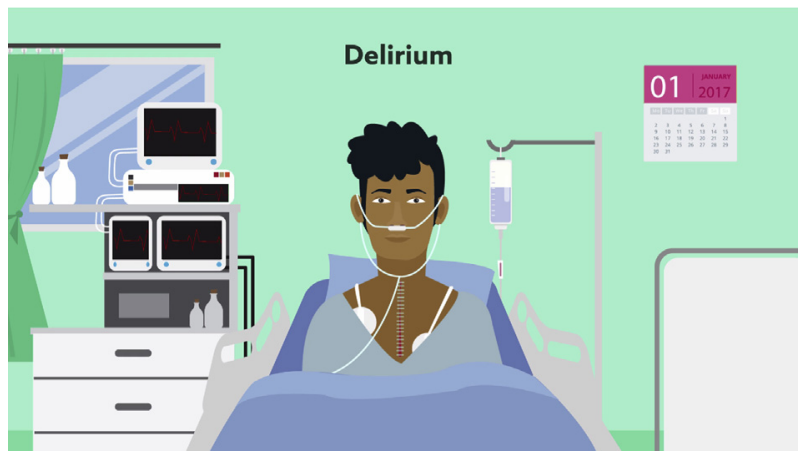

VIDEO 1. Delirium increases risk of fast-track failure, regardless of subtype. Video available at: http://www.jtcvsonline.org/article/S00225223(17)32141-4/fulltext.

We examined motor subtypes categorized by CAM-ICU assessment and RASS scores (Figure 2). The distribution of mixed delirium across RASS scores suggests that mixed delirium is a category that probably reflects changes in sedation-agitation shifts, partly from the effects of pharmacologic agents used in postoperative care. Notably, pharmacologic agents were more likely to be prescribed to our patients with mixed delirium than with pure hyperactive delirium or pure hypoactive delirium. This is not surprising given the physical manifestation of hypoactivity. Because the hypoactive delirium subtype is associated with outcomes no better than other subtypes, future studies to establish therapeutic strategies to treat delirium should not ignore the need to effectively recognize and treat hypoactive delirium. ${ }^{15}$

Targeted therapies for hypoactive delirium and early light sedation in mixed and hyperactive subtypes may reduce the incidence, onset, and severity of delirium by careful titration of analgo-sedative agents to avoid sudden major fluctuations in sedation levels. ${ }^{34,35}$ Apart from pharmacologic therapies (eg, dexmedetomidine, quetiapine), nonpharmacologic therapies (eg, early mobilization, sleep promotion, family engagement) should be considered. The findings of this study suggest that such strategies should be applied to all subtypes, including the diagnostically challenging hypoactive subtype.

\section{Study Limitations}

There are several limitations to this study. First, the incidence of hypoactive and mixed delirium is possibly an underestimate due to misclassification bias because we did not measure delirium occurring in the ward after ICU discharge. Therefore, our reported RRs may be an underestimate. Second, we did not use a validated delirium severity tool, such as the ICU-7 delirium severity scale that is based on the CAM-ICU and RASS ${ }^{33}$ because this tool was not available when the study commenced. We used a previous tool ${ }^{23}$ that summed each delirium episode (absolute RASS score plus 1 point) during the observation period in the ICU to show greater delirium severity. The strengths of the study include meticulous and complete data collection, efforts to minimize performance bias in RASS and CAM-ICU assessments from bedside nurses who were unaware of the study objectives, rigorous risk adjustment, and the use of lag-time approach to address the issue of protopathic bias (reverse causation). ${ }^{31}$

\section{CONCLUSIONS}

Delirium is a major complication after cardiac surgery. This study showed an association between delirium and FTF. After risk factor adjustment, patients with hypoactive delirium were at similar risk of developing FTF or prolonged length of stay as other motor subtypes. Differentiation of motor subtypes is unlikely to be clinically important for prognostication in early recovery after cardiac surgery programs. However, because all types of delirium are associated with adverse outcomes, potential management strategies should address the diagnosis and treatment of all individual subtypes (Video 1).

\section{Conflict of Interest Statement}

Authors have nothing to disclose with regard to commercial support.

The authors thank the staff involved in CAM-ICU assessment training before study commencement.

\section{References}

1. Wong WT, Lai VK, Chee YE, Lee A. Fast-track cardiac care for adult cardiac surgical patients. Cochrane Database Syst Rev. 2016;9:CD003587.

2. Fleming IO, Garratt C, Guha R, Desai J, Chaubey S, Wang Y, et al. Aggregation of marginal gains in cardiac surgery: feasibility of a perioperative care bundle for enhanced recovery in cardiac surgical patients. J Cardiothorac Vasc Anesth. 2016;30:665-70.

3. Lee A, Zhu F, Underwood MJ, Gomersall CD. Fast-track failure after cardiac surgery: external model validation and implications to ICU bed utilization. Crit Care Med. 2013;41:1205-13.

4. Kogan A, Ghosh P, Preisman S, Tager S, Sternik L, Lavee J, et al. Risk factors for failed "fast-tracking" after cardiac surgery in patients older than 70 years. J Cardiothorac Vasc Anesth. 2008;22:530-5.

5. Waseem Z, Lindner J, Sgouropoulou S, Eibel S, Probst S, Scholz M, et al. Independent risk factors for fast-track failure using a predefined fast-track protocol in preselected cardiac surgery patients. J Cardiothorac Vasc Anesth. 2015;29:1461-5.

6. Youssefi P, Timbrell D, Valencia O, Gregory P, Vlachou C, Jahangiri M, et al. Predictors of failure in fast-track cardiac surgery. $J$ Cardiothorac Vasc Anesth. 2015;29:1466-71.

7. Kiessling AH, Huneke P, Reyher C, Bingold T, Zierer A, Moritz A. Risk factor analysis for fast track protocol failure. J Cardiothorac Surg. 2013;8:47.

8. Gleason LJ, Schmitt EM, Kosar CM, Tabloski P, Saczynski JS, Robinson T, et al. Effect of delirium and other major complications on outcomes after elective surgery in older adults. JAMA Surg. 2015;150:1134-40.

9. Breu A, Stransky M, Metterlein T, Werner T, Trabold B. Subsyndromal delirium after cardiac surgery. Scand Cardiovasc J. 2015;49:207-12.

10. Katznelson R, Djaiani GN, Borger MA, Friedman Z, Abbey SE, Fedorko L, et al. Preoperative use of statins is associated with reduced early delirium rates after cardiac surgery. Anesthesiology. 2009;110:67-73.

11. Rudolph JL, Jones RN, Levkoff SE, Rockett C, Inouye SK, Sellke FW, et al. Derivation and validation of a preoperative prediction rule for delirium after cardiac surgery. Circulation. 2009;119:229-36. 
12. Brown CH IV, Laflam A, Max L, Lymar D, Neufeld KJ, Tian J, et al. The impact of delirium after cardiac surgical procedures on postoperative resource use. Ann Thorac Surg. 2016;101:1663-9.

13. Koster S, Hensens AG, van der Palen J. The long-term cognitive and functional outcomes of postoperative delirium after cardiac surgery. Ann Thorac Surg. 2009;87:1469-74.

14. Saczynski JS, Marcantonio ER, Quach L, Fong TG, Gross A, Inouye SK, et al. Cognitive trajectories after postoperative delirium. N Engl J Med. 2012;367: 30-9.

15. Meagher D. Motor subtypes of delirium: past, present and future. Int Rev Psychiatry. 2009;21:59-73.

16. Robinson TN, Raeburn CD, Tran ZV, Brenner LA, Moss M. Motor subtypes of postoperative delirium in older adults. Arch Surg. 2011;146:295-300.

17. Zhang W, Hu W, Shen M, Ye X, Huang Y, Sun Y. Profiles of delirium and the clinical outcomes of patients who underwent coronary artery bypass grafting: a prospective study from China. J Clin Nurs. 2016;25:631-41.

18. Stransky M, Schmidt C, Ganslmeier P, Grossmann E, Haneya A, Moritz S, et al. Hypoactive delirium after cardiac surgery as an independent risk factor for prolonged mechanical ventilation. J Cardiothorac Vasc Anesth. 2011;25: 968-74.

19. Lee A, Mu JL, Joynt GM, Chiu CH, Lai VKW, Gin T, et al. Risk prediction models for delirium in the intensive care unit after cardiac surgery: a systematic review and independent external validation. Br J Anaesth. 2017;118:391-9.

20. Sessler CN, Gosnell MS, Grap MJ, Brophy GM, O’Neal PV, Keane KA, et al. The Richmond Agitation-Sedation Scale: validity and reliability in adult intensive care unit patients. Am J Respir Crit Care Med. 2002;166:1338-44.

21. Ely EW, Margolin R, Francis J, May L, Truman B, Dittus R, et al. Evaluation of delirium in critically ill patients: validation of the Confusion Assessment Method for the Intensive Care Unit (CAM-ICU). Crit Care Med. 2001;29:1370-9.

22. Pandharipande P, Cotton BA, Shintani A, Thompson J, Costabile S, Truman Pun B, et al. Motoric subtypes of delirium in mechanically ventilated surgical and trauma intensive care unit patients. Intensive Care Med. 2007;33:1726-31.

23. Milbrandt EB, Deppen S, Harrison PL, Shintani AK, Speroff T, Stiles RA, et al. Costs associated with delirium in mechanically ventilated patients. Crit Care Med. 2004;32:955-62.
24. Colantuoni E, Dinglas VD, Ely EW, Hopkins RO, Needham DM. Statistical methods for evaluating delirium in the ICU. Lancet Respir Med. 2016;4:534-6.

25. Constantinides VA, Tekkis PP, Fazil A, Kaur K, Leonard R, Platt M, et al Fast-track failure after cardiac surgery: development of a prediction model Crit Care Med. 2006;34:2875-82.

26. Mascha EJ, Sessler DI. Statistical grand rounds: design and analysis of studies with binary- event composite endpoints: guidelines for anesthesia research. Anesth Analg. 2011;112:1461-71.

27. Shrier I, Platt RW. Reducing bias through directed acyclic graphs. BMC Med Res Methodol. 2008;8:70.

28. Textor J, Hardt J, Knuppel S. DAGitty: a graphical tool for analyzing causal diagrams. Epidemiology. 2011;22:745.

29. Heinze G, Schemper M. A solution to the problem of separation in logistic regression. Stat Med. 2002;21:2409-19.

30. Hosmer DW, Lemeshow S, Sturdivant RX. Applied Logistic Regression. 3rd ed Hoboken: John Wiley \& Sons; 2013.

31. Arfe A, Corrao G. The lag-time approach improved drug-outcome association estimates in presence of protopathic bias. J Clin Epidemiol. 2016;78:101-7.

32. van den Boogaard M, Schoonhoven L, van der Hoeven JG, van Achterberg T, Pickkers P. Incidence and short-term consequences of delirium in critically ill patients: a prospective observational cohort study. Int J Nurs Stud. 2012;49: 775-83.

33. Khan BA, Perkins AJ, Gao S, Hui SL, Campbell NL, Farber MO, et al. The Confusion Assessment Method for the ICU-7 Delirium Severity Scale: a nove delirium severity instrument for use in the ICU. Crit Care Med. 2017;45:851-7.

34. Svenningsen H, Egerod I, Videbech P, Christensen D, Frydenberg M Tonnesen EK. Fluctuations in sedation levels may contribute to delirium in ICU patients. Acta Anaesthesiol Scand. 2013;57:288-93.

35. Barr J, Fraser GL, Puntillo K, Ely EW, Gélinas C, Dasta JF, et al. Clinical practice guidelines for the management of pain, agitation, and delirium in adult patients in the intensive care unit. Crit Care Med. 2013;41:263-306.

Key Words: cardiac surgical procedures, delirium, postoperative complications 


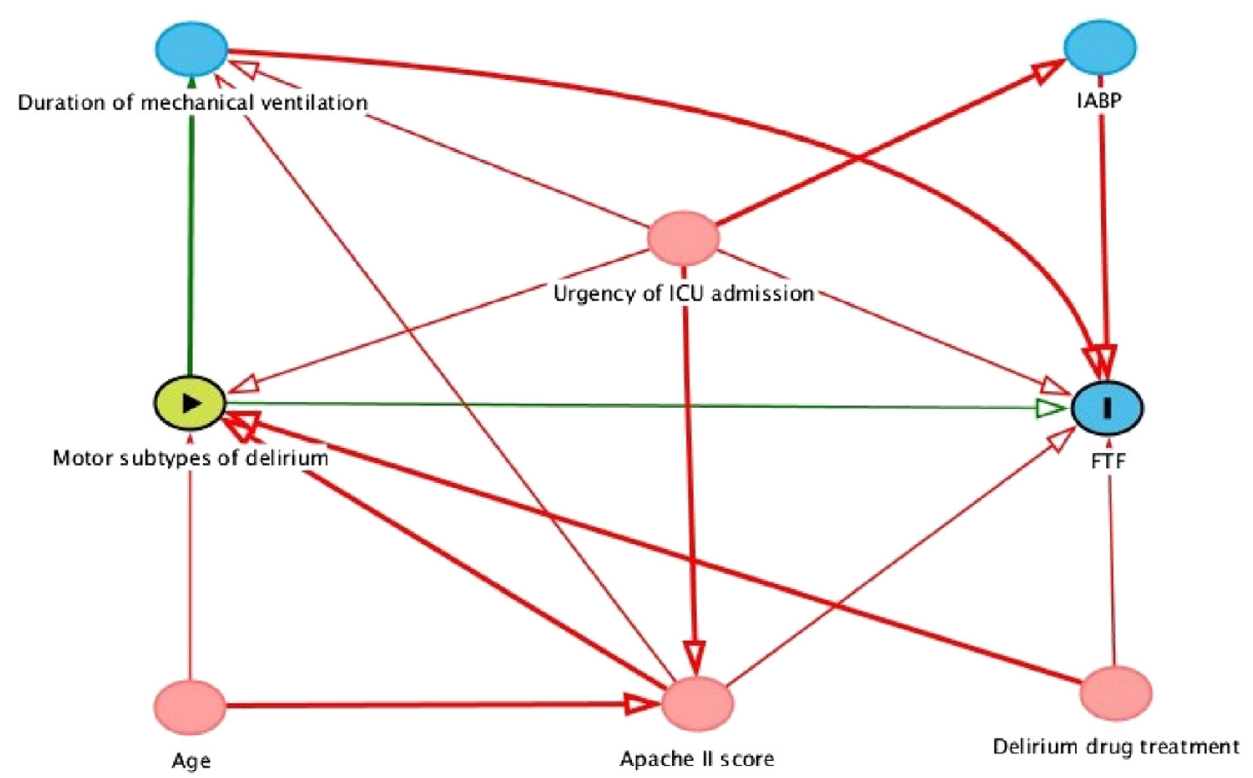

FIGURE E1. Directed acyclic graph. Assumptions made in the motor subtypes of delirium (exposure) and FTF (outcome) relationship to identify the set of variables needed for confounding adjustment. Green circle represents exposure. Blue circle represents ancestor of outcome. Red circle represents ancestor of exposure and outcome. All red arrows lie on open biasing paths. All green arrows lie on open causal paths. Bold arrow indicate no corresponding indirect path (no causal effect between variables exists if arrow is removed). Thin arrow indicates there is another indirect pathway between variables. The total effect is the main analysis chosen because we are interested in the overall or total effect of motor subtypes on FTF. This total effect is divided into the direct effect of motor subtypes of delirium and indirect (or mediated) effects. The direct effect of motor subtypes on FTF, or the effect per se of motor subtypes, is adjusted for by the duration of mechanical ventilation. The indirect effect of motor subtypes of delirium on FTF occurs by first affecting the mediator, duration of mechanical ventilation, which in turn causes an increased risk of FTF. The mediator, duration of mechanical ventilation, lies on the causal pathway between motor subtypes of delirium and FTF. In contrast, confounders (age, APACHE II score, urgency of ICU admission, delirium drug treatment) are associated with motor subtypes of delirium and causally related to FTF. ICU, Intensive care unit; IABP, intra-aortic balloon pump; FTF, fast-track failure; $A P A C H E$, Acute Physiology and Chronic Health Evaluation. 